\title{
Emodin-induced autophagy against cell apoptosis through the PI3K/AKT/mTOR pathway in human hepatocytes
}

This article was published in the following Dove Press journal:

Drug Design, Development and Therapy

\author{
Xiao-yuan Zheng' \\ Shi-ming Yang ${ }^{2}$ \\ Rong Zhang' \\ Su-min Wang ${ }^{2}$ \\ Guo-bing Li' \\ Shi-wen Zhou' \\ 'Department of Pharmacy, Xinqiao \\ Hospital, Army Medical University, \\ Chongqing, People's Republic of China; \\ ${ }^{2}$ Department of Gastroenterology, \\ Xinqiao Hospital, Army Medical \\ University, Chongqing, People's Republic \\ of China
}

Correspondence: Shi-wen Zhou Department of Pharmacy, Xinqiao Hospital, Army Medical University, Chongqing 400037, People's Republic of China

Email zhouswxinqiao@I26.com
Background: Emodin, a major component of Polygonum multiflorum (PM), has been reported to exert both protective and toxic effects in several cell types. However, the effects and underlying mechanisms of action of emodin in hepatic cells are still obscure.

Methods: The present study used the normal human liver cell line L02 to investigate the effects and mechanisms of emodin in hepatic cells. After treatment with emodin, L02 cells were examined for viability, apoptosis and autophagy with the Cell Counting Kit-8 (CCK-8), annexin V/PerCP staining and GFP-LC3 plasmid transfection. The expression of proteins including cleaved caspase-3, LC3B-I/II, p-PI3K, PI3K, p-AKT, AKT, p-mTOR, mTOR and actin was examined by using Western blot.

Results: Emodin significantly inhibited the viability of and induced apoptosis in L02 cells in a dose- and time-dependent manner. In addition, emodin increased the number of GFP-LC3 puncta in L02 cells and upregulated the expression of LC3B-II compared to those in control cells. Furthermore, emodin significantly decreased the expression of p-PI3K, p-AKT and pmTOR in a dose-dependent manner compared to that in control cells without altering the expression of PI3K, AKT and mTOR. Notably, cotreatment with emodin and 3-methyladenine (3-MA) or rapamycin significantly increased and decreased the apoptosis rate of L02 cells, respectively, compared to that of cells treated with emodin alone.

Conclusion: In conclusion, emodin exhibited cytotoxicity in the L02 human hepatic cell line by promoting apoptosis, and it also induced autophagy through the suppression of the $\mathrm{PI} 3 \mathrm{~K} / \mathrm{AKT} / \mathrm{mTOR}$ signalling pathway. The autophagy could play a protective role following emodin treatment.

Keywords: Polygonum multiflorum, emodin, hepatoprotective, apoptosis, hepatotoxic, autophagy, PI3K, AKT, mTOR

\section{Introduction}

Polygonum multiflorum is a traditional Chinese herb that has been widely used as an anti-ageing treatment in China for centuries, but it has recently been reported to cause liver injury. ${ }^{1,2}$ Anthraquinones, stilbenes and tannins are thought to be the major active components of $P M{ }^{3}$ Emodin is an anthraquinone derivative (Figure 1A) isolated from $P M$. Emodin exhibits a variety of therapeutic effects, such as anti-tumour, ${ }^{4}$ antioxidant, ${ }^{5}$ anti-inflammatory, ${ }^{6}$ and anti-virus activities. ${ }^{7}$ Despite its various biological properties, both protective activities and toxic effects were reported with emodin treatment in vitro and in vivo. ${ }^{8,9}$ Thus, for its safe clinical use, the underlying mechanism of action of emodin in hepatic cells still needs to be elucidated. 
A<smiles>CCOCCO</smiles>

B

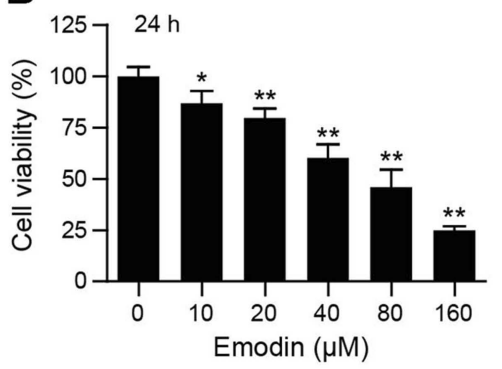

C

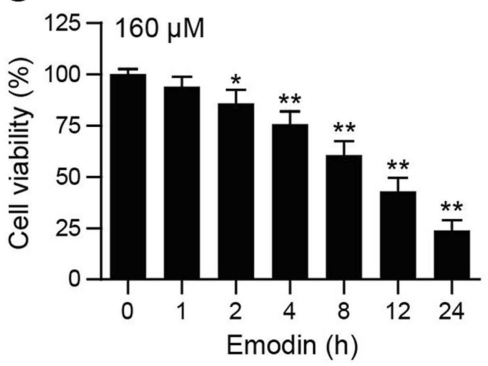

D

Emodin $(\mu \mathrm{M})$



Figure I Emodin suppressed the proliferation of L02 cells. (A) The chemical structure of emodin. (B and C) L02 cells were treated with different concentrations of emodin for $24 \mathrm{~h}$ or with emodin $(160 \mu \mathrm{M})$ for different time intervals. Cell viability was determined by CCK-8 assay. Data are presented as the means \pm SDs for 3 independent experiments. *P<0.05 compared with the control group; $* * P<0.01$ compared with the control group. (D) Representative photos depict the morphology of L02 cells exposed to various concentrations of emodin for $24 \mathrm{~h}$. Scale bars: $100 \mu \mathrm{m}$.

Macroautophagy (hereafter, autophagy) is a catabolic process that degrades cytoplasmic components within the lysosome and provides additional nutrients and energy to the cell. ${ }^{10}$ In addition, autophagy plays an essential role in quality control by selectively degrading long-lived proteins and organelles. Because of its anti-stress properties, autophagy is commonly thought to be an essential cytoprotective response to several stresses, including starvation, hypoxia and pathogen invasion. Conversely, it has also been reported that excessive autophagy in cells may promote caspase- and apoptosis-independent cell death. ${ }^{11}$ The dynamic process of autophagy is tightly regulated. At the initiation of autophagy, most signal pathways converge to the same mammalian target, rapamycin complex 1 (mTORC1, a complex containing TOR, raptor, G $\beta \mathrm{L} /$ mLST8, PRAS40 and DEPTOR), especially mTOR. ${ }^{12,13}$ MTOR is a serine/threonine protein kinase belonging to the phosphatidylinositol kinase-related kinase family. The activity of mTOR is suppressed under nutrient starvation, which induces autophagy. The phosphatidylinositol 3kinase (PI3K)/AKT pathway is a pathway upstream of mTOR that plays a central role in cell survival and proliferation. Previous studies showed that emodin exerted a pro-apoptotic effect on K562 cells by suppressing the PI3K/AKT signalling pathway. ${ }^{14}$ Controversially, emodin attenuated autophagy in mouse $\mathrm{C} 2 \mathrm{C} 12$ myoblasts through the phosphorylation of AKT. ${ }^{15}$ These results pose the questions of whether emodin had a role in autophagic activity in hepatic cells and what the mechanism of emodin treatment in hepatocytes was.

In this study, we investigated the effects and molecular mechanisms of emodin on L02 hepatic cells and sought to understand the hepatoprotective activities and hepatotoxic effects of emodin treatment. Our data demonstrated that emodin can inhibit L02 cell proliferation, trigger cell apoptosis, and induce autophagy in L02 cells through the $\mathrm{PI} 3 \mathrm{~K} / \mathrm{AKT} / \mathrm{mTOR}$ pathway. These results provide evidence for the rational use of emodin in the future.

\section{Materials and methods \\ Cell lines and reagents}

L02 cells were purchased from the Cell Bank of the Chinese Academy of Sciences and maintained in RPMI 1640 medium (Gibco, 31800022) supplemented with $10 \%$ foetal bovine serum (FBS; Gibco, 10100147), $1.5 \mathrm{~g} / \mathrm{L} \mathrm{NaHCO}_{3}$ (Gibco, 25080094), and $0.11 \mathrm{~g} / \mathrm{L}$ sodium pyruvate (Gibco, 11360070 ) in $5 \% \mathrm{CO}_{2}$ at $37^{\circ} \mathrm{C}$. Emodin (E7881), 3-methyladenine (M9281) and rapamycin (V900930) were purchased from Sigma-Aldrich Chemical. The antibodies used were as follows: anti-cleaved caspase-3 (\#9664), anti-LC3B-I/II (\#3868), anti-phospho-AKT(Thr308) (\#13038), anti-AKT (\#4691), anti-phospho-mTOR(Ser2448) (\#5536), anti-mTOR (\#2983), and anti-actin (\#8457), which were purchased from 
Cell Signalling Technology. Anti-phospho-PI3K(Tyr485) (sc130211) and anti-PI3K (sc-365404) were purchased from Santa Cruz Biotechnology. Horseradish peroxidase-conjugated species-specific secondary antibodies (4741506, 4741806) were purchased from Kirkegaard and Perry Laboratories.

\section{Cell viability (CCK-8) assay}

Cells were seeded in 96-well plates at a density of 5,000 cells per well for $24 \mathrm{~h}$, and 5 identical wells were prepared for each group. After the indicated treatments, the cells were treated with $10 \mu \mathrm{l}$ of Cell Counting Kit- 8 reagent (CCK-8) (Beyotime, C0038) for $1 \mathrm{~h}$. The absorption values (optical density (OD)) were measured by a microplate reader at $450 \mathrm{~nm}$. Cell viabilities were normalized to the control group according to the following formula: cell viability $(\%)=\left(\mathrm{OD}_{\text {blank }}-\mathrm{OD}_{\text {experiment }}\right) / \mathrm{OD}_{\text {blank }} \times 100 \%$.

\section{Cell morphology}

To view the cell morphology, L02 cells were seeded in 6well plates at a density of $5 \times 10^{4}$ cells per well for $24 \mathrm{~h}$ and treated with various concentrations of emodin for $24 \mathrm{~h}$. The morphology of the L02 cells was observed under a light microscope (Diaphot 300, Nikon).

\section{GFP-LC3 plasmid transfection and confocal microscopy}

A total of $2 \times 10^{4}$ cells were plated on coverslips for $24 \mathrm{~h}$. GFP-LC3 plasmid transfection was performed using Lipofectamine 3000 transfection reagent (Invitrogen, L3000) according to the manufacturer's protocol. Plasmids encoding GFP-LC3 (Addgene, \#22405) were a gift from Jayanta Debnath. After a $48 \mathrm{~h}$ incubation, the medium containing the transfection mixture was replaced with fresh complete medium and the cells were treated with emodin $(80 \mu \mathrm{M})$ for $12 \mathrm{~h}$ or were not treated with emodin before being viewed by using a laser scanning confocal microscope (Zeiss, Germany). All images were analysed by ImageJ software (MD, USA).

\section{Flow cytometry}

After the cells were treated as indicated, the apoptosis rates were determined by flow cytometry using an Annexin V Apoptosis Detection Kit PerCP-eFluor (eBioscience, 88-8008-74) according to the manufacturer's instructions.

\section{Western blots}

The cells were treated as indicated, harvested and then washed twice with ice-cold PBS for total protein extraction. The protein concentration was determined using an Enhanced BCA Protein Assay Kit (Beyotime Biotechnology, P0011, China). Equal amounts of protein were separated by $10 \%$ SDS-PAGE and subsequently transferred onto polyvinylidene difluoride (PVDF) membranes. The membranes were blocked with 5\% skim milk in TBST buffer (20 mM Tris-HCl, pH 7.4, $150 \mathrm{mM} \mathrm{NaCl}$, and $0.1 \%$ Tween 20 ). Then, the membranes were incubated with primary antibodies at $4{ }^{\circ} \mathrm{C}$ overnight. After that, the membranes were washed 3 times with TBST buffer and incubated with the secondary antibodies at room temperature for $2 \mathrm{~h}$. After the membranes were washed with TBST buffer 3 times, bands were observed and analysed with a charge-coupled device (CCD) system (Kodak, Image Station 2000 MM, Japan).

\section{Statistical analysis}

Statistical analyses were performed with SAS. Data were normalized to the control and analysed with onesample $t$-tests. Statistical significance was indicated as follows: $0.01 \leq * P<0.05 ; * * P<0.01$.

\section{Results}

\section{Emodin suppressed the proliferation of L02 cells}

To observe the inhibitory effects of emodin on hepatic cells, we first performed CCK-8 assays to investigate the effects of emodin on L02 cell growth. As shown in Figure 1B and C, emodin decreased the viability of L02 cells in a dose- and time-dependent manner. To directly observe alterations to L02 cells with emodin treatment, we viewed cells under an optical microscope with a camera. As shown in Figure 1D, the untreated L02 cells grew well, and the cellular structures were clear. After treatment with emodin for $24 \mathrm{~h}$, we found increased cellular shrinkage and decreased cell density in a dosedependent manner.

\section{Emodin induced apoptosis in LO2 cells through the activation of caspase- 3}

To examine the inhibitory effects of emodin on L02 cells, we assessed the effects of emodin on apoptosis in L02 cells by using an annexin V/PerCP staining assay. Cells treated with emodin exhibited increased apoptosis in a 


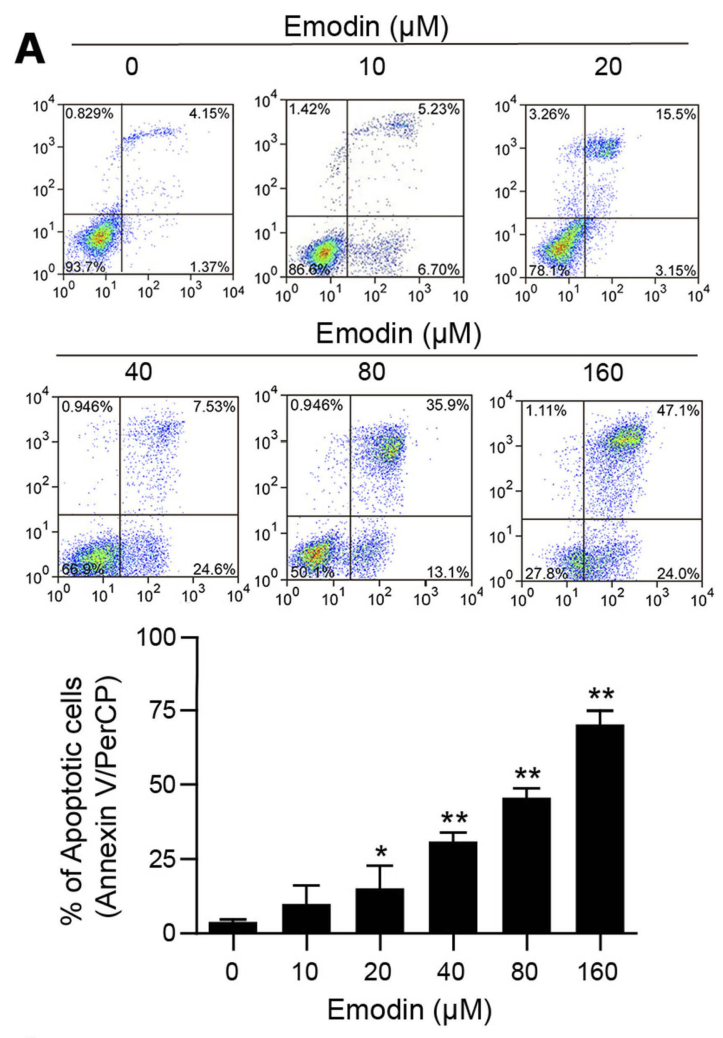

C

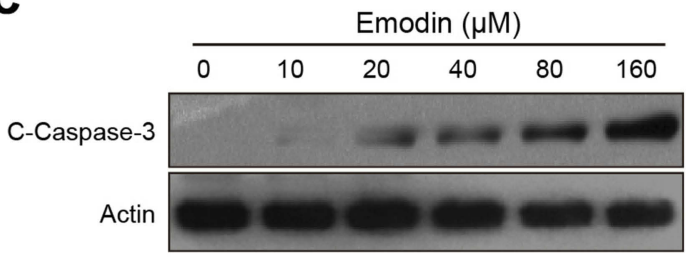

E



B
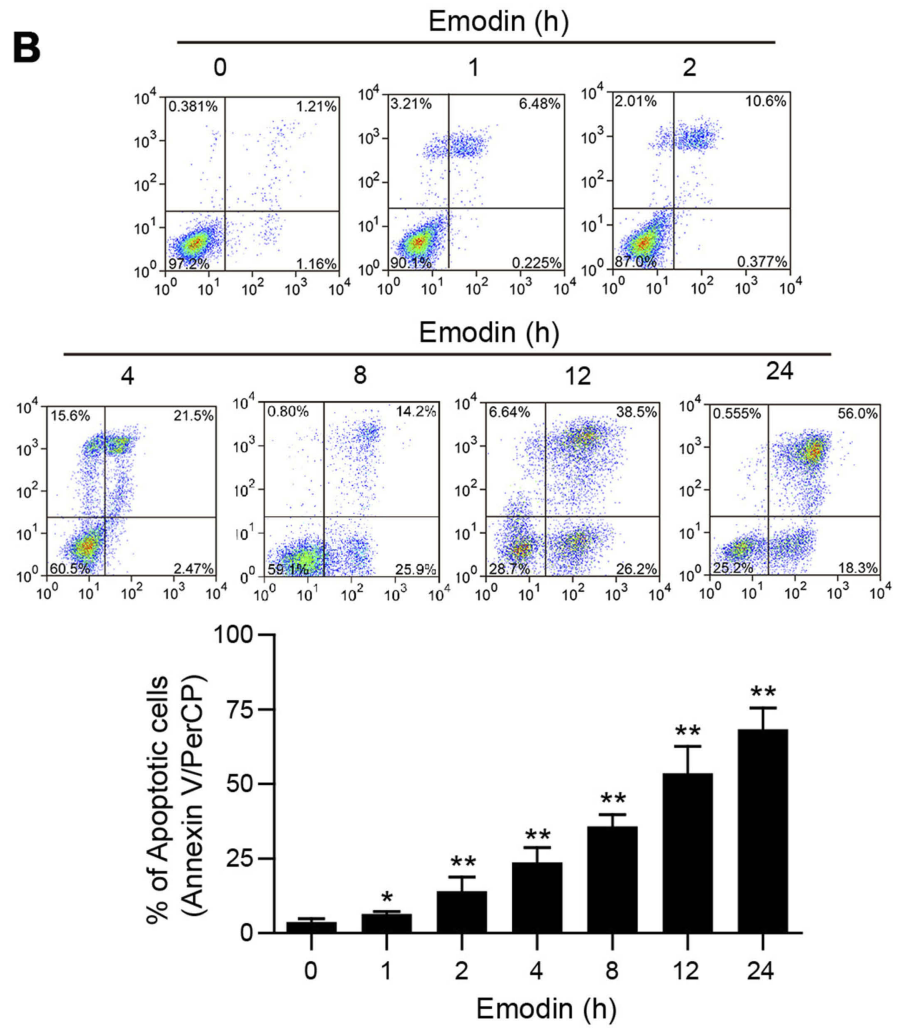

D

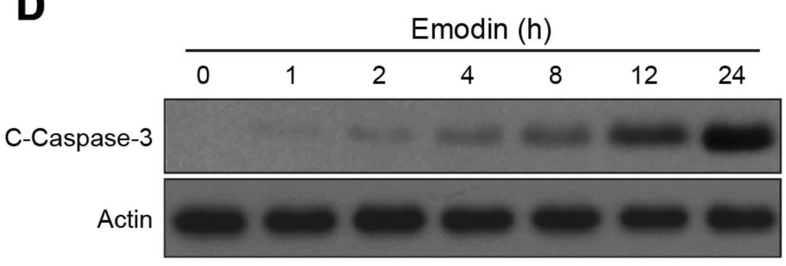

F

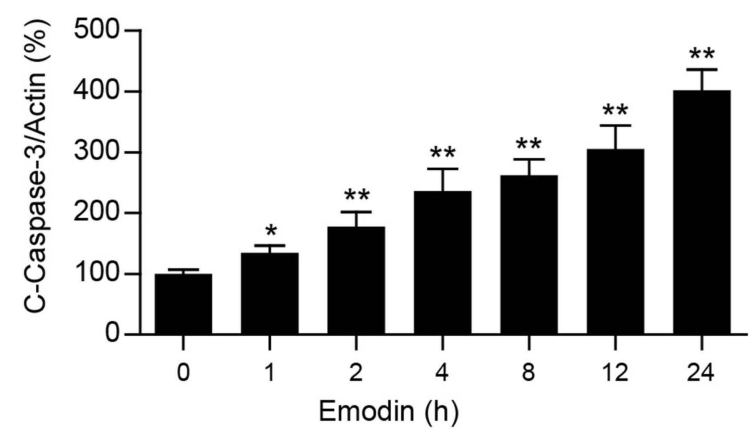

Figure 2 Emodin induced apoptosis in L02 cells through activating caspase-3. (A and B) L02 cells were treated with different concentrations of emodin for $24 \mathrm{~h}$ or with $160 \mu \mathrm{M}$ emodin for different time intervals. Cell apoptosis was detected by flow cytometry using annexin V/PerCP staining. Representative flow cytometric images and statistical data are showed, and the data are presented as means \pm SDs for 3 independent experiments. $* P<0.05$ compared with the control group; $* * P<0.0$ l compared with the control group. (C and D) Cells were treated as in (A) and (B), respectively, and then subjected to Western blot analysis to determine the expression of c-caspase-3. Actin was used as a loading control. (E) The intensities of c-caspase-3 in (C) and (D) normalized to actin were statistically analysed and represented as the mean \pm SD for 3 independent experiments. $* P<0.05$ compared with the control group; $* * P<0.01$ compared with the control group. (F) The intensities of $c$-caspase- 3 in (D) normalized to actin were statistically analysed and represented as the mean \pm SD for 3 independent experiments. $* P<0.05$ compared with the control group; $* * P<0.01$ compared with the control group.

dose- and time-dependent manner (Figure 2A and B). In addition to the increase in apoptosis, following emodin treatment, the expression of cleavage/activation caspase-3 (c-caspase-3) was increased in a dose- and time-dependent manner compared to that observed without emodin treatment (Figure 2C-F). These results indicated that emodin 
could induce apoptosis in L02 cells by activating caspase-3.

\section{Emodin induced autophagic alterations in LO2 cells}

Whether emodin plays a role in the autophagic activity of L02 cells remains unclear. Because the conversion of LC3B-I to LC3B-II is commonly used to evaluate autophagy, ${ }^{16}$ we first detected LC3B-I/II levels in L02 cells using Western blotting. As shown in Figure 3A-D, emodin treatment unexpectedly led to an increase in the expression of LC3B-II in a dose- and time-dependent manner, except for $160 \mu \mathrm{M}$ emodin treatment for $24 \mathrm{~h}$, and the expression of LC3B-I was largely unchanged compared to that in the untreated cells. Furthermore, L02 cells expressing GFP-LC3 were detected under a confocal laser scanning microscope used to observe autophagosomes. The treatment of cells with emodin $(80 \mu \mathrm{M})$ for $12 \mathrm{~h}$ resulted
A

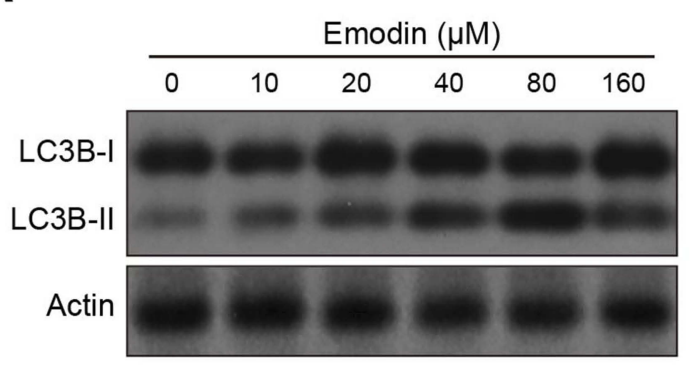

C

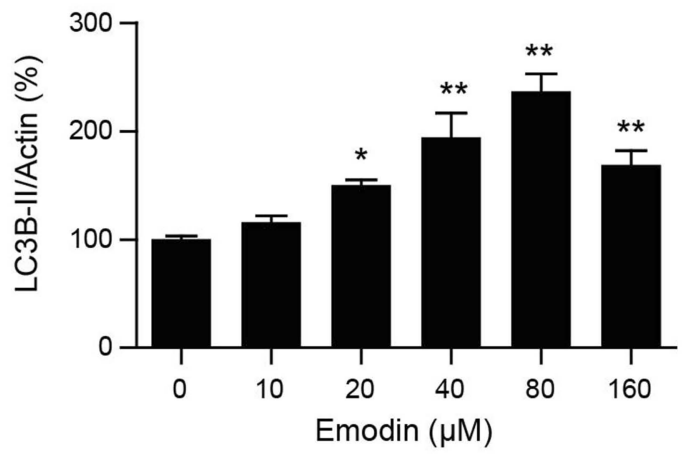

B

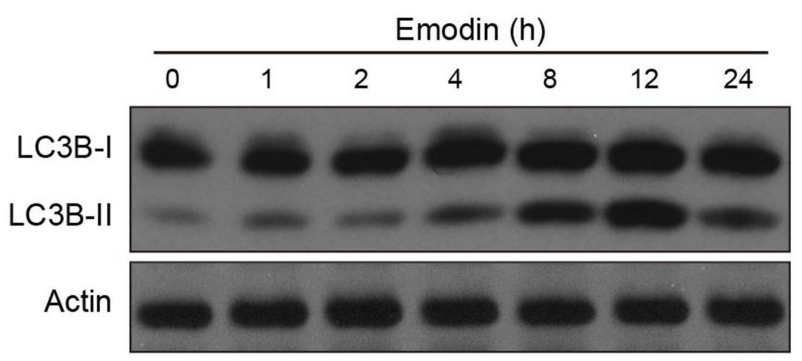

D

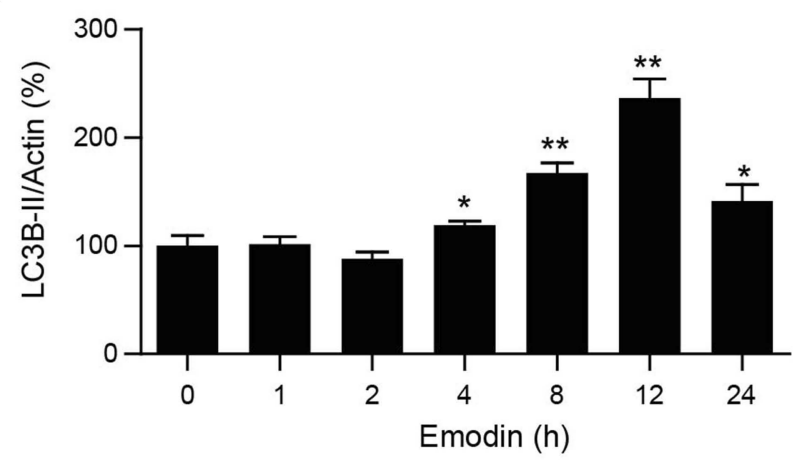

E

Control

Emodin $(80 \mu \mathrm{M})$

$\mathbf{F}$
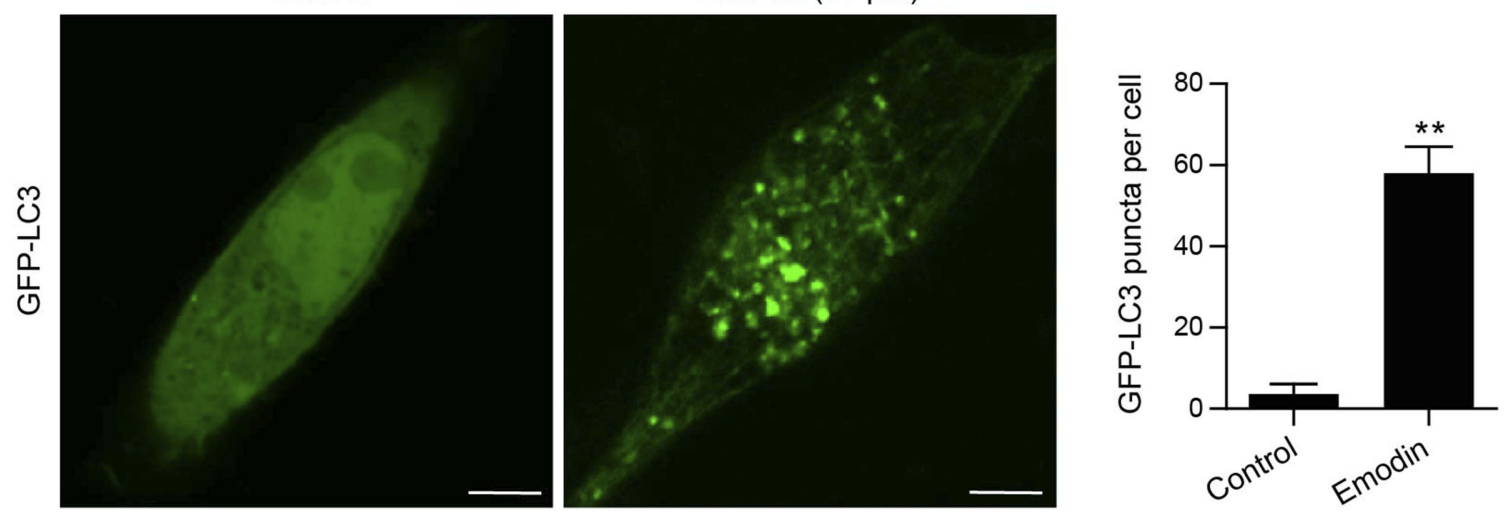

Scale bar 10 um

Figure 3 Emodin induced autophagic alterations in L02 cells. (A and B) Cells were treated with different concentrations of emodin for $24 \mathrm{~h}$ or with emodin (I60 $\mu \mathrm{M})$ for different time intervals. The expression of LC3B-I/II was analysed by Western blot. (C and D) The intensities of LC3B-II in (A and B) normalized to actin were statistically analysed and represented as the mean \pm SD for 3 independent experiments. $* P<0.05$ compared with the control group; $* * P<0.0$ I compared with the control group. (E) GFPLC3 expression in L02 cells treated with or without emodin $(80 \mu \mathrm{M})$ for $12 \mathrm{~h}$. GFP-LC3 puncta were examined using confocal microscopy; scale bars: $10 \mu \mathrm{m}$. (F) The average number of GFP-LC3 puncta per cell in (E) was quantitated from 3 independent experiments. Data are presented as the mean \pm SD for 3 independent experiments. Thirty cells were analysed per treatment condition. $* * 00.01$ compared with the control group. 
in a significant increase in GFP-LC3 puncta formation compared to that in untreated cells, which indicated the accumulation of autophagosomes (Figure 3E and F).

\section{Emodin inhibited the PI3K/AKT/mTOR pathway}

To further understand the mechanism of emodin in autophagy, the levels of p-PI3K, PI3K, p-AKT, AKT, p-mTOR and mTOR were detected. Western blot analysis showed that emodin treatment resulted in a dose-dependent decrease in the expression of p-PI3K, p-AKT and p-mTOR in L02 cells compared to that observed in cells without emodin treatment; however, the level of p-mTOR was marginally elevated with $10 \mu \mathrm{M}$ emodin treatment compared to that observed in cells without emodin treatment (Figure 4A and B). In addition, changes in the expression levels of PI3K, AKT and mTOR were not observed (Figure $4 \mathrm{~A}$ and $\mathrm{B}$ ). These results suggested that emodin might induce autophagy through the suppression of the PI3K/AKT/mTOR pathway.

\section{Emodin-induced autophagy played a protective role in $\mathrm{LO2}$ cells}

Autophagy has been reported to be both a protective and a lethal process in cells, so we investigated the role of emodin-induced autophagy by using 3-MA, an autophagy inhibitor that targets the initiation of autophagy. ${ }^{17}$ Cotreatment of L02 cells with emodin and 3-MA significantly reduced the expression of LC3B-II and cell viability compared with those in cells treated with emodin alone (Figure 5A-C). In addition, the apoptosis rates were significantly increased with emodin and 3-MA treatment compared with those after treatment with emodin alone (Figure 5D). The L02 cells were largely shrinkage with apoptotic morphologies under cotreatment with emodin and 3-MA (Figure 5E). Moreover, cotreatment of L02 cells with rapamycin (an autophagy inducer that targets mTOR) and emodin significantly increased the expression of LC3B-II and cell viability compared with those after treatment with emodin alone (Figure 5F-H) and markedly attenuated apoptosis compared with the apoptosis rates following treatment with emodin alone (Figure 5I). The L02 cells exhibited reduced shrinkage with rapamycin and emodin cotreatment than with emodin alone (Figure 5J). These data suggested that emodin-induced autophagy had a protective role in $\mathrm{L} 02$ cells.

\section{Discussion}

In recent years, the role of traditional Chinese herbs in the treatment of multiple diseases has drawn much attention from scientists because of their high efficiencies and low toxicities. Emodin, an anthraquinone derivative isolated from Polygonum multiflorum, has gained increasing attention as a therapeutic agent. Leukaemia, cervical cancer, lung cancer, colorectal cancer and hepatocellular carcinoma cells were reported to be targets of emodin, with less toxicity for non-tumour cells reported in previous studies. ${ }^{18-22}$ Recently, the hepatotoxicity of emodin was reported to be $\sim 30 \mu \mathrm{M}^{23}$ Consistent with this report, we found that emodin at concentrations above $20 \mu \mathrm{M}$ had an obvious cytotoxic effect on the hepatic cell line L02 by promoting cell apoptosis. In addition to its anti-tumour activities, emodin protected against diabetic cardiomyopathy by regulating the AKT/GSK-3 $\beta$ signalling pathway, ${ }^{24}$ and it was
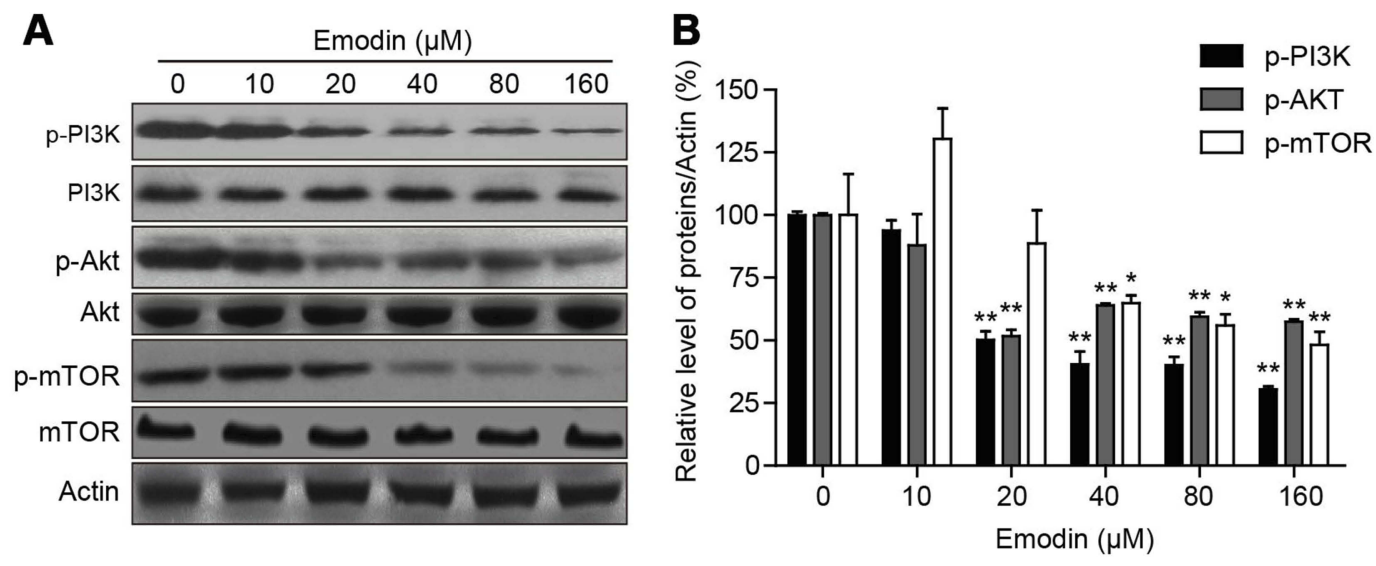

Figure 4 Emodin inhibited the PI3K/AKT/mTOR pathway. (A) L02 cells were treated with different concentrations of emodin for 24 h. The expression of $p-P I 3 K$ (Tyr485), PI3K, p-AKT (Thr308), AKT, p-mTOR (Ser2448) and mTOR were analysed by Western blot. (B) The intensities of p-PI3K, p-AKT and p-mTOR in (A) normalized to actin were statistically analysed and represented as the mean \pm SD for 3 independent experiments. $* P<0.05$ compared with the control group; $* * P<0.01$ compared with the control group. 
A

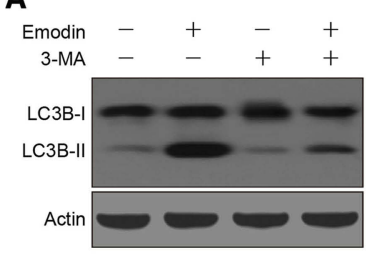

C

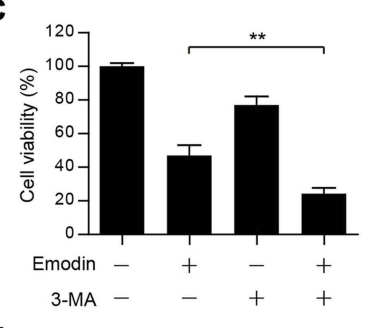

E

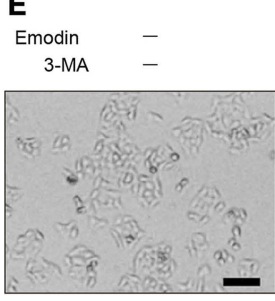

F

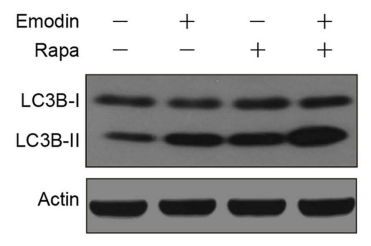

H

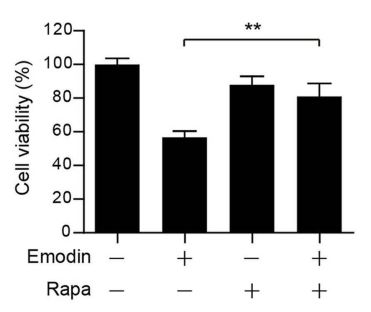

B

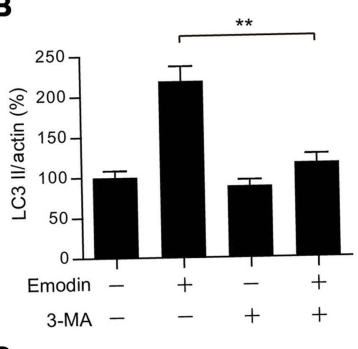

D

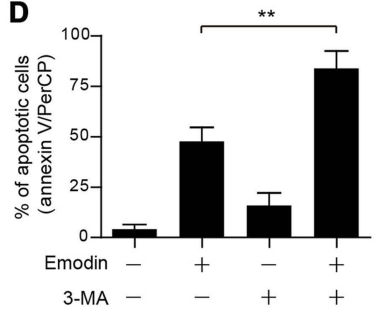

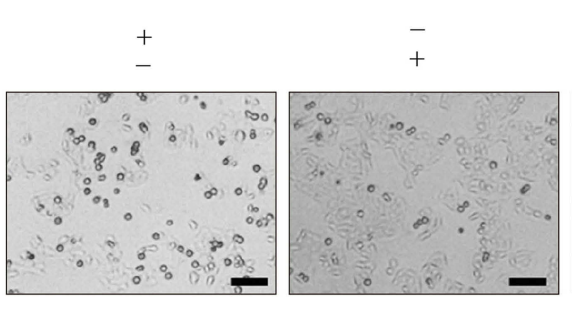

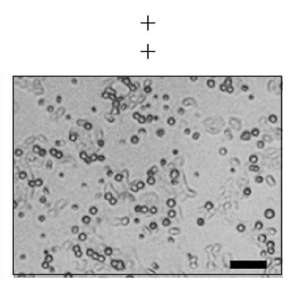

scale bar 100um

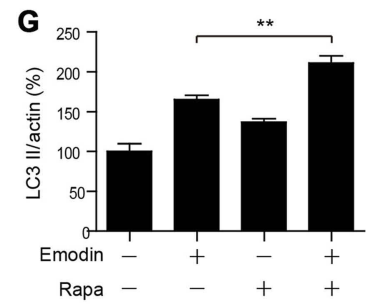

I
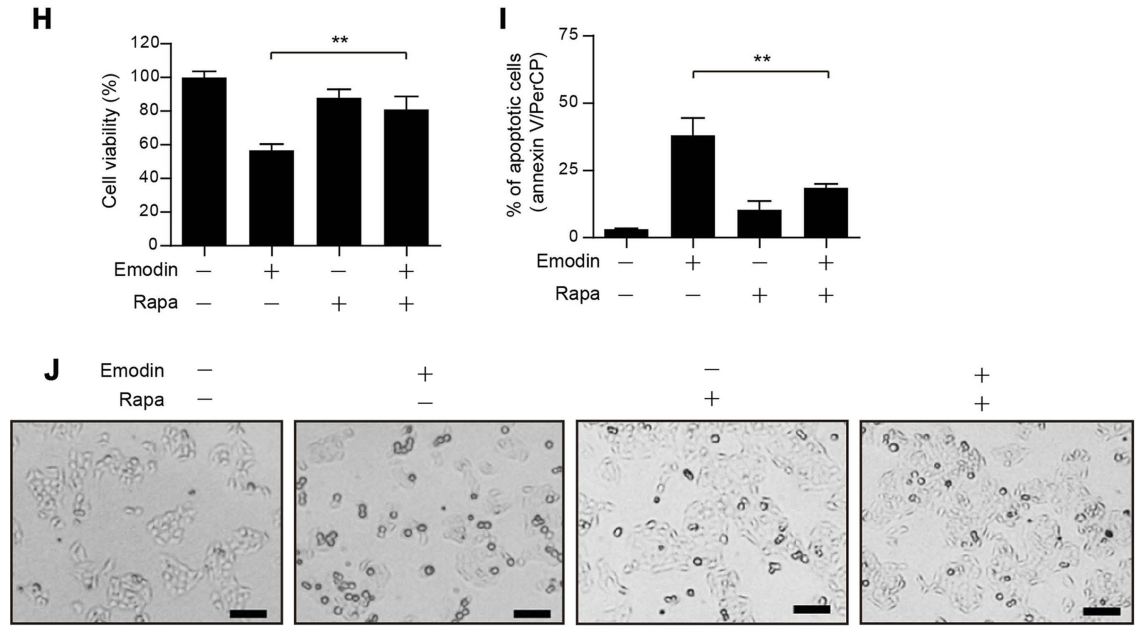

Scale bar 100um

Figure 5 Emodin-induced autophagy played a protective role in L02 cells. (A) Cells were treated with or without emodin $(40 \mu M)$ in the absence or presence of 3-MA (5 mM) for $24 \mathrm{~h}$. The expression of LC3B-I/II was analysed by Western blot. (B) The intensities of LC3B-II in (A) normalized to actin were statistically analysed and represented as the mean \pm SD for 3 independent experiments. **P<0.0I. (C) Cell viability in (A) was determined by CCK-8 assay. Data are presented as the means \pm SDs for 3 independent experiments. ${ }^{* * P}<0.01$. (D) Cell apoptosis in (A) was detected by flow cytometry using annexin V/PerCP staining. Data are presented as the means \pm SDs for 3 independent experiments. (E) Representative photos depicting the morphology of L02 cells treated in (A) Scale bars: $100 \mu \mathrm{m}$. (F) Cells were treated with or without emodin $(40 \mu \mathrm{M})$ in the absence or presence of rapamycin (Rapa; $100 \mathrm{nM}$ ) for $24 \mathrm{~h}$. The expression of LC3B-I/II was analysed by Western blot. (G) The intensities of LC3B-Il in (F) normalized to actin were statistically analysed and are represented as the mean \pm SD for 3 independent experiments. $* * P<0.01$. (H) Cell viability in $(\mathbf{F})$ was determined by CCK-8 assay. Data are presented as the means \pm SDs for 3 independent experiments. **P<0.0I. (I) Cell apoptosis in $(\mathbf{F})$ was detected by flow cytometry using annexin V/PerCP staining. Data are presented as the means \pm SDs for 3 independent experiments. (J) Representative photos depicting the morphology of L02 cells treated in (F). Scale bars: $100 \mu \mathrm{m}$. 
also reported that emodin protected against cisplatin-induced apoptosis in rat renal tubular cells by activating autophagy through the modulation of the AMPK/mTOR pathway. ${ }^{25}$ Consistent with these reports, we also found that emodin could induce autophagy in the L02 hepatic cell line with the following evidence: (a) emodin treatment significantly upregulated the expression of LC3B-II in a dose- and time-dependent manner compared to that observed without emodin treatment and resulted in the significant accumulation of autophagosomes in L02 cells; (b) emodin caused the significant downregulation of the expression of $\mathrm{p}-\mathrm{PI} 3 \mathrm{~K}, \mathrm{p}-\mathrm{AKT}$ and $\mathrm{p}$ mTOR without any obvious changes in the expression of PI3K, AKT and $\mathrm{mTOR}$ in a dose-dependent manner compared to that observed without emodin treatment; and (c) the cotreatment of cells with emodin and 3-MA reduced the expression of LC3BII, and the combinational treatment of cells with emodin (40 $\mu \mathrm{M}$, a non-saturating concentration that induces autophagy) and rapamycin enhanced the expression of LC3B-II compared to that observed following treatment with emodin alone.

Autophagy is commonly considered an anti-apoptotic process that maintains the health of mammalian cells, probably by eliminating damaged organelles such as mitochondria, ${ }^{26}$ the endoplasmic reticulum, ${ }^{27}$ and part of the nucleus. ${ }^{28}$ In addition, autophagy is also thought to be a kind of programmed cell death with excessive engulfment and degradation of the cytoplasm, although the mechanism of this is still largely unclear, and this classification is still controversial. ${ }^{29}$ In the present study, both the autophagy inhibitor 3-MA and the autophagy inducer rapamycin were recruited to understand the role of emodin-induced autophagy in hepatocytes. The combination of emodin with 3-MA significantly promoted apoptosis in L02 cells compared to that observed in cells treated with emodin alone. Furthermore, emodin and rapamycin cotreatment markedly attenuated apoptosis in L02 cells compared with that observed following emodin treatment alone. These results suggested that emodin-induced autophagy has a protective role against cellular apoptosis, especially in at low/medium dose $(10-80 \mu \mathrm{M})$. Notably, with a high dose $(160 \mu \mathrm{M})$ of emodin, the expression of LC3B-II was reduced compared to that observed with $80 \mu \mathrm{M}$ emodin treatment. This could partly be due to the caspase-mediated cleavage of essential autophagy proteins, which produced conditions in which autophagy could not cope with cellular stresses. ${ }^{30}$ Additionally, the protective effect of emodin-induced autophagy in hepatic cells could be reinforced by rapamycin, illustrating that mTOR might be a potential therapeutic target for

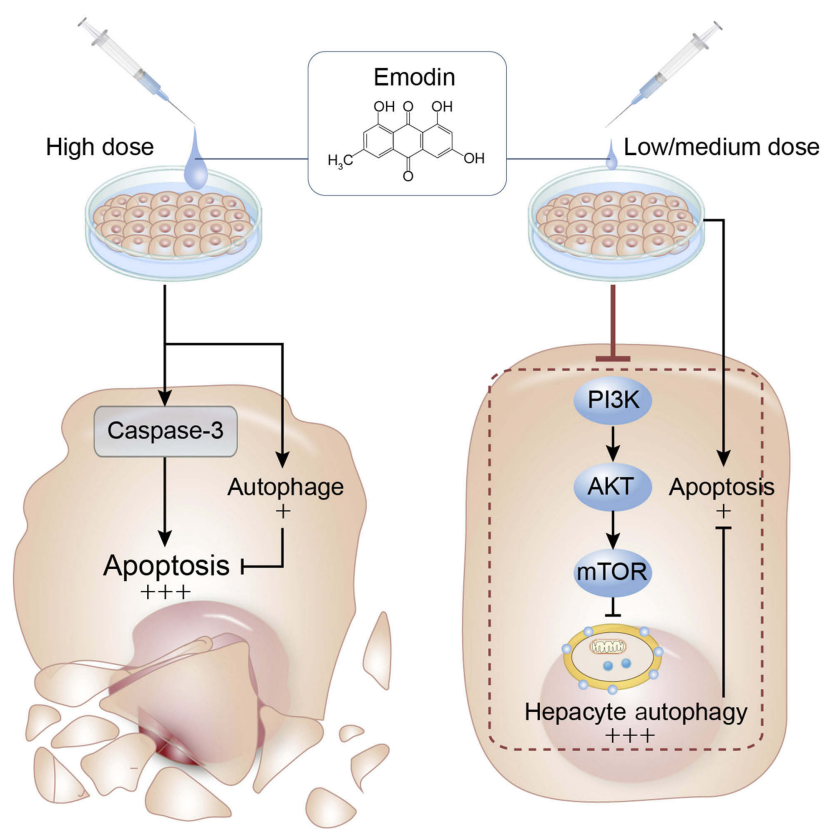

Figure 6 Summary of the current study. At low/medium concentrations, emodininduced autophagy hinders apoptosis and maintains LO2 cell viability, while high concentrations of emodin induces a marginal autophagy which hardly cope apoptosis and leads cell death. Arrows and black words part represent activation of target molecules or stimulation of production, and blunt lines and grey words part indicate target inhibition.

liver protection in the future. More attention needs to be paid to emodin for its future clinical usage because, although emodin showed hepatoprotective effects in some reports, it exerted hepatotoxic effects in a dose- and time-dependent manner in our present study.

In summary, we demonstrated that emodin induced L02 cell apoptosis through upregulating the expression of cleaved caspase- 3 and induced L02 cells autophagy through attenuating the phosphorylation of PI3K, AKT and mTOR. Autophagy could play a protective role against cell apoptosis in hepatocytes (Figure 6). These findings improved the understanding of the hepatoprotective activities and hepatotoxic effects of emodin treatment.

\section{Abbreviation list}

3-MA, 3-methyladenine; AMPK, adenosine 5'-monophosphate (AMP)-activated protein kinase; DEPTOR, DEP domain-containing mTOR-interacting protein; GFP, green fluorescent protein; LC3, microtubule-associated protein 1A/1B-light chain 3; PRAS40, proline-rich AKT substrate of $40 \mathrm{kDa}$; SDS-PAGE, sodium dodecyl sulfate-polyacrylamide gel electrophoresis. 


\section{Acknowledgments}

This work was partially supported by the Natural Science Foundation of Chongqing (cstc2018jscx-msyX0172), and the authors would like to thank the Central Laboratory of Xinqiao Hospital at the Army Medical University.

\section{Author contributions}

The study was conceived and designed by Shi-wen Zhou, Shi-ming Yang and Xiao-yuan Zheng. Technical support was provided by Rong Zhang, Guo-bing Li and Su-min Wang. The manuscript was drafted by Xiao-yuan Zheng and revised by Shi-wen Zhou and Shi-ming Yang. All authors contributed to data analysis, drafting or revising the article, gave final approval of the version to be published, and agree to be accountable for all aspects of the work.

\section{Disclosure}

The authors report no conflicts of interest in this work.

\section{References}

1. Park GJH, Mann SP, Ngu MC. Acute hepatitis induced by Shou-WuPian, a herbal product derived from polygonum multiflorum. $J$ Gastroenterol Hepatol. 2001;16(1):115-117.

2. Mazzanti G, Battinelli L, Daniele C, et al. New case of acute hepatitis following the consumption of Shou Wu Pian, a Chinese herbal product derived from Polygonum multiflorum. Ann Intern Med. 2004;140:7. doi:10.7326/0003-4819-140-7-200404060-00042-w3

3. Ma J, Zheng L, He YS, Li HJ. Hepatotoxic assessment of polygoni multiflori radix extract and toxicokinetic study of stilbene glucoside and anthraquinones in rats. $J$ Ethnopharmacol. 2015;162:61-68. doi:10.1016/j.jep.2014.12.045

4. Shrimali D, Shanmugam MK, Kumar AP, et al. Targeted abrogation of diverse signal transduction cascades by emodin for the treatment of inflammatory disorders and cancer. Cancer Lett. 2013;341(2):139149. doi:10.1016/j.canlet.2013.08.023

5. Ahmad W, Zaidi SMA, Mujeeb M, Ansari SH, Ahmad S. HPLC and HPTLC methods by design for quantitative characterization and in vitro anti-oxidant activity of polyherbal formulation containing rheum emodi. J Chromatogr Sci. 2014;52(8):911-918. doi:10.1093/chromsci/bmt123

6. Chang C-H, Lin -C-C, Yang -J-J, Namba T, Hattori M. Anti-inflammatory effects of emodin from ventilago leiocarpa. Am J Chin Med (Gard City N Y). 1996;24(02):139-142. doi:10.1142/S0192415X96000189

7. Zhang HM, Wang F, Qiu Y, et al. Emodin inhibits coxsackievirus B3 replication via multiple signalling cascades leading to suppression of translation. Biochem J. 2016;473(4):473-485. doi:10.1042/ BJ20150419

8. Yin X, Gong X, Jiang R, et al. Emodin ameliorated lipopolysaccharideinduced fulminant hepatic failure by blockade of TLR4/MD2 complex expression in D-galactosamine-sensitized mice. Int Immunopharmacol. 2014;23(1):66-72. doi:10.1016/j.intimp.2014.08.018

9. Liu X, Liu Y, Qu Y, Cheng M, Xiao H. Metabolomic profiling of emodin-induced cytotoxicity in human liver cells and mechanistic study. Toxicol Res (Camb). 2015;4(4):948-955. doi:10.1039/ C4TX00246F

10. Green DR, Levine B. To be or not to be? How selective autophagy and cell death govern cell fate. Cell. 2014;157(1):65-75. doi:10.1016/ j.cell.2014.02.049
11. Denton D, Nicolson S, Kumar S. Cell death by autophagy: facts and apparent artefacts. Cell Death Differ. 2012;19(1):87-95. doi:10.1038/ cdd.2011.146

12. Jung CH, Ro SH, Cao J, Otto NM, Kim DH. mTOR regulation of autophagy. FEBS Lett. 2010;584(7):1287-1295. doi:10.1016/j. febslet.2010.01.017

13. Laplante M, Sabatini DM. mTOR signaling in growth control and disease. Cell. 2012;149(2):274-293. doi:10.1016/j.cell.2012.03.017

14. Chun-Guang W, Liang Z, Yong LL, et al. Emodin exerts an antiapoptotic effect on human chronic myelocytic leukemia K562 cell lines by targeting the PTEN/PI3K-AKT signaling pathway and deleting BCR-ABL. Integr Cancer Ther. 2016;16(4):526-539. doi: $10.1177 / 1534735416664784$

15. Chen D, Liu J, Lu L, et al. Emodin attenuates TNF-alpha-induced apoptosis and autophagy in mouse $\mathrm{C} 2 \mathrm{C} 12$ myoblasts though the phosphorylation of Akt. Int Immunopharmacol. 2016;34:107-113. doi:10.1016/j.intimp.2016.02.023

16. Klionsky DJ, Abdelmohsen K, Abe A, et al. Guidelines for the use and interpretation of assays for monitoring autophagy (3rd edition). Autophagy. 2016;12(1):1-222.

17. Li X, Xu HL, Liu YX, An N, Zhao S, Bao JK. Autophagy modulation as a target for anticancer drug discovery. Acta Pharmacol Sin. 2013;34(5):612-624. doi:10.1038/aps.2013.23

18. Pooja T, Karunagaran D. Emodin suppresses Wnt signaling in human colorectal cancer cells SW480 and SW620. Eur J Pharmacol. 2014;742:55-64. doi:10.1016/j.ejphar.2014.08.028

19. Yaoxian W, Hui Y, Yunyan Z, Yanqin L, Xin G, Xiaoke W. Emodin induces apoptosis of human cervical cancer hela cells via intrinsic mitochondrial and extrinsic death receptor pathway. Cancer Cell Int. 2013;13(1):1. doi:10.1186/1475-2867-13-71

20. Yu JQ, Bao W, Lei JC. Emodin regulates apoptotic pathway in human liver cancer cells. Phytother Res. 2013;27(2):251-257. doi:10.1002/ptr.4703

21. Ok S, Kim S-M, Kim C, et al. Emodin inhibits invasion and migration of prostate and lung cancer cells by downregulating the expression of chemokine receptor CXCR4. Immunopharmacol Immunotoxicol. 2012;34(5):768-778. doi:10.3109/08923973.2012.654494

22. Chen Y, Li J, Hu J, et al. Emodin enhances ATRA-induced differentiation and induces apoptosis in acute myeloid leukemia cells. Int $J$ Oncol. 2014;45(5):2076-2084. doi:10.3892/ijo.2014.2610

23. Li CL, Ma J, Zheng L, Li HJ, Li P. Determination of emodin in L-02 cells and cell culture media with liquid chromatography-mass spectrometry: application to a cellular toxicokinetic study. J Pharm Biomed Anal. 2012;71:71-78. doi:10.1016/j.jpba.2012.07.031

24. Wu Z, Chen Q, Ke D, Li G, Deng W. Emodin protects against diabetic cardiomyopathy by regulating the AKT/GSK-3beta signaling pathway in the rat model. Molecules. 2014;19(9):14782-14793. doi:10.3390/molecules190914782

25. Liu H, Gu LB, Tu Y, Hu H, Huang YR, Sun W. Emodin ameliorates cisplatin-induced apoptosis of rat renal tubular cells in vitro by activating autophagy. Acta Pharmacol Sin. 2016;37(2):235-245. doi:10.1038/aps.2015.114

26. Li Q, Zhang T, Wang J, et al. Rapamycin attenuates mitochondrial dysfunction via activation of mitophagy in experimental ischemic stroke. Biochem Biophys Res Commun. 2014;444(2):182-188. doi:10.1016/j.bbrc.2014.01.032

27. Lipatova Z, Segev N, Hochstrasser M. A role for macro-ER-phagy in ER quality control. PLoS Genet. 2015;11(7):e1005390. doi:10.1371/ journal.pgen. 1005390

28. Dou Z, Xu C, Donahue G, et al. Autophagy mediates degradation of nuclear lamina. Nature. 2015;527(7576):105. doi:10.1038/nature15724

29. Kroemer G, Levine B. Autophagic cell death: the story of a misnomer. Nat Rev Mol Cell Biol. 2008;9(12):1004-1010. doi:10.1038/ nrm2529

30. Marino G, Niso-Santano M, Baehrecke EH, Kroemer G. Self-consumption: the interplay of autophagy and apoptosis. Nat Rev Mol Cell Biol. 2014;15(2):81-94. doi:10.1038/nrm3735 


\section{Publish your work in this journal}

Drug Design, Development and Therapy is an international, peerreviewed open-access journal that spans the spectrum of drug design and development through to clinical applications. Clinical outcomes, patient safety, and programs for the development and effective, safe, and sustained use of medicines are a feature of the journal, which has also been accepted for indexing on PubMed Central. The manuscript management system is completely online and includes a very quick and fair peer-review system, which is all easy to use. Visit http://www. dovepress.com/testimonials.php to read real quotes from published authors. 